\title{
93.
}

\section{NOTE SUR QUELQUES FORMULES QUI SE RAPPORTENT À LA MULTIPLICATION DES FONCTIONS ELLIPTIQUES.}

[From the Journal für die reine und angewandte Mathematik (Crelle), tom. xxxIx. (1850), pp. 16-22.]

LES fonctions

$\{\lambda, \mu, x, y\}=P_{0,0}-\left(\frac{1}{1} P_{1,0} x+\frac{1}{1} P_{0,1} y\right)+\left(\frac{1}{1.2} P_{2,0} x^{2}+\frac{1}{1.1} P_{1,1} x y+\frac{1}{1.2} P_{0,2} y^{2}\right)-\& c$.

où $P_{0,0}=1$ et les autres coefficients sont donnés par l'équation à différences

$$
\begin{gathered}
{\left[-l \lambda-m \mu+(l-m)^{2}\right] P_{l, m}} \\
+l(\lambda-2 l+2 m+2)(\lambda-2 l+2 m+1) P_{l-1, m} \\
+m(\mu+2 l-2 m+2)(\mu+2 l-2 m+1) P_{l, m-1} \\
-16 l m[\lambda \mu-(2 l+2 m-4)(\lambda+\mu)] P_{l-1, m-1}=0
\end{gathered}
$$

jouent, comme je crois, un rôle important dans la théorie des fonctions elliptiques ${ }^{\text {. }}$

1 La fonction $\{\lambda, \mu, x, y\}$ satisfait à l'équation

$$
\begin{aligned}
& -[\lambda(\lambda-1) x+\mu(\mu-1) y+16 \lambda \mu x y] u \\
& +[-(\lambda-1)+(4 \lambda-6) x+(4 \mu+2) y+32(\lambda+\mu) x y] y \frac{d u}{d x} \\
& +[-(\mu-1)+(4 \lambda+2) x+(4 \mu-6) y+32(\lambda+\mu) x y] y \frac{d u}{d y} \\
& +(1-4 x-4 y)\left(x^{2} \frac{d^{2} u}{d x^{2}}-2 x y \frac{d^{2} u}{d x d y}+y^{2} \frac{d^{2} u}{d y^{2}}\right)=0,
\end{aligned}
$$

qui peut être tirée de l'equation

$$
n(n-1) x^{2} u+(n-1)\left(\alpha x-2 x^{3}\right) \frac{d u}{d x}+\left(1-a x^{2}+x^{4}\right) \frac{d^{2} u}{d x^{2}}-2 n\left(a^{2}-4\right) \frac{d u}{d a}=0
$$

(voyez le mémoire cité plus bas), mais qu'on obtient plus facilement au moyen de l'équation à différences à laquelle satisfont les coefficients $P_{l, m}$. 
En effet, en faisant $x=\sqrt{ } k \sin$ am $u, \alpha=k+\frac{1}{k}$ et en représentant par $z$ le dénominateur de la fonction $\sqrt{ } k \sin$ am $n u$ (où $n$ est un entier positif quelconque), on aura

$$
z=z_{1}+z_{2}+\ldots+z_{8}+\ldots,
$$

cette série étant continuée jusqu'au terme $z_{\frac{1}{2} n}$ ou $z_{\frac{1}{2}(n-1)}$, selon que $n$ est pair ou impair, et la fonction $z$ étant donnée par l'équation

$$
z_{s}=(-1)^{(n+1) s}(4 \alpha)^{s(n-2 s)} x^{2 n s}\left\{n^{2}-2 n s, 2 n s, \frac{x^{2}}{4 \alpha}, \frac{1}{4 \alpha x^{2}}\right\},
$$

où cependant les termes qui contiennent des puissances négatives de $\alpha$ doivent être négligés. Ces formules reviennent à celles que j'ai présentées dans la "Note sur les fonctions elliptiques" (t. xxxvII.), [67].

En revenant aux fonctions $\{\lambda, \mu, x, y\}$, j'ai trouvé les deux formules

$$
\begin{aligned}
P_{l, 0} & =\lambda[\lambda-l-1]^{l-1}, \\
P_{l, 1} & =\mu \lambda[\lambda-l-1]^{l-1} \\
& +l \lambda[\lambda-l-1]^{l-3}\left\{(18 l-16) \lambda-\left(16 \lambda^{2}-10 l-4\right)\right\} \\
& -\frac{10 l \lambda \mu}{\lambda+\mu} \lambda[\lambda-l]^{l-2}
\end{aligned}
$$

(où selon la notation de Vandermonde la factorielle $p(p-1) \ldots(p-q+1)$ est exprimée par $\left.[p]^{q}\right)$. De là, et en calculant la valeur de $P_{2,2}$ à l'aide de l'équation à différences, on obtient:

$$
\begin{aligned}
& P_{0,0}=1, \\
& P_{1,0}=\lambda, \\
& P_{0,1}=\mu, \\
& P_{2,0}=\lambda(\lambda-3), \\
& P_{1,1}=\left(\lambda \mu+2-\frac{10 \lambda \mu}{\lambda+\mu}\right) \\
& P_{0,2}=\mu(\mu-3) \\
& P_{3,0}=\lambda(\lambda-4)(\lambda-5), \\
& P_{2,1}=\lambda\left((\lambda-3) \mu+40-\frac{20 \lambda \mu}{\lambda+\mu}\right), \\
& P_{1,2}=\mu\left((\mu-3) \lambda+40-\frac{20 \lambda \mu}{\lambda+\mu}\right), \\
& P_{0,3}=\mu(\mu-4)(\mu-5),
\end{aligned}
$$




$$
\begin{aligned}
& P_{4,0}=\lambda(\lambda-5)(\lambda-6)(\lambda-7), \\
& P_{3,1}=\lambda\left(\mu(\lambda-4)(\lambda-5)+114 \lambda-330-\frac{30 \lambda \mu}{\lambda+\mu}(\lambda-3)\right), \\
& P_{2,2}=\left(\lambda(\lambda-3) \mu(\mu-3)+152 \lambda \mu+336-\frac{40 \lambda^{2} \mu^{2}+1156 \lambda \mu}{\lambda+\mu}+\frac{200 \lambda^{2} \mu^{2}}{(\lambda+\mu)^{2}}\right), \\
& P_{1,3}=\mu\left(\lambda(\mu-4)(\mu-5)+114 \mu-330-\frac{30 \lambda \mu}{\lambda+\mu}(\mu-3)\right), \\
& P_{0,4}=\mu(\mu-5)(\mu-6)(\mu-7)
\end{aligned}
$$

la première partie de cette table se trouve dans la note citée.

Nous remarquerons en passant que pour $y=0$, on a $\{\lambda, \mu, x, 0\}=\left(\frac{1}{2}+\sqrt{ }\left(\frac{1}{4}-x\right)\right)^{\lambda}$. On sait que la théorie de la multiplication des fonctions circulaires depend de la fonction $\left(x+\sqrt{ }\left(x^{2}-1\right)\right)^{\lambda}$ ou, en faisant $\frac{1}{4} x^{-2}=x$, de la fonction $\left(\frac{1}{2}+\sqrt{ }\left(\frac{1}{4}-x\right)\right)^{\lambda}$. Cela fait espérer que l'on parviendra par les fonctions $\{\lambda, \mu, x, y\}$ à la théorie complète de la multiplication des fonctions elliptiques.

J'ai calculé les valeurs qui servent à trouver les dénominateurs $z$ de $\sin$ am $n u$, où $n$ est un quelconque des entiers $1,2,3,4,5,6,7$. Les voici:

$$
\begin{aligned}
& n=1, \quad z_{0}=1, \quad z=z_{0}, \\
& n=2, \quad z_{0}=1, \quad-z_{1}=-x^{4}, \quad z=z_{0}-z_{1}, \\
& n=3, \quad z_{0}=1, \quad+z_{1}=4 \alpha x^{6}-\left(6 x^{4}+3 x^{8}\right), \quad z=z_{0}+z_{1}, \\
& n=4, \quad z_{0}=1, \quad-z_{1}=-16 \alpha^{2} x^{8}+4 \alpha\left(8 x^{10}+8 x^{6}\right)-\left(20 x^{12}+26 x^{8}+20 x^{4}\right), \\
& z_{2}=x^{16}, \quad z=z_{0}-z_{1}+z_{2}, \\
& n=5, \quad z_{0}=1 \text {, } \\
& z=z_{0}+z_{1}+z_{2} \\
& z_{1}=64 \alpha^{3} \quad x^{10} \\
& -16 \alpha^{2}\left(15 x^{12}+10 x^{8}\right) \\
& +4 \alpha\left(90 x^{14}+92 x^{10}+35 x^{6}\right) \\
& -\quad\left(275 x^{16}+300 x^{12}+125 x^{8}+50 x^{4}\right) \text {, } \\
& z_{2}=16 \alpha^{2} \quad x^{20} \\
& -4 \alpha\left(5 x^{22}+20 x^{18}\right) \\
& +\quad\left(5 x^{24}+62 x^{20}+170 x^{26}\right), \\
& n=6, \quad+z_{0}=1 \text {, } \\
& z=z_{0}-z_{1}+z_{2}-z_{3}, \\
& -z_{1}=-256 \alpha^{4} \quad x^{12} \\
& +64 \alpha^{3}\left(24 x^{14}+12 x^{10}\right) \\
& -16 \alpha^{2}\left(252 x^{16}+210 x^{12}+54 x^{8}\right) \\
& +\quad 4 \alpha\left(1520 x^{18}+1584 x^{14}+576 x^{10}+112 x^{6}\right) \\
& -\quad\left(5814 x^{20}+7704 x^{16}+2400 x^{12}+444 x^{8}+105 x^{4}\right) \text {, }
\end{aligned}
$$




$$
\begin{aligned}
& +z_{2}=256 \alpha^{4} \quad x^{24} \\
& \text { - } 64 \alpha^{3}\left(12 x^{26}+24 x^{22}\right) \\
& +16 a^{2}\left(54 x^{28}+210 x^{24}+252 x^{20}\right) \\
& -4 \alpha\left(112 x^{30}+576 x^{26}+1584 x^{22}+1520 x^{18}\right) \\
& +\quad\left(105 x^{32}+444 x^{28}+2400 x^{24}+7704 x^{20}+5814 x^{16}\right), \\
& -z_{3}=\quad-x^{36} \\
& n=7 \text {, } \\
& z_{0}=1 \text {, } \\
& z_{1}=1024 \alpha^{5} \quad x^{14} \\
& -256 \alpha^{4}\left(35 x^{16}+\quad 14 x^{12}\right) \\
& +64 \alpha^{3}\left(1120 x^{18}+196 x^{14}+\quad 77 x^{10}\right) \\
& -16 \alpha^{2}\left(5425 x^{20}+5040 x^{16}+1575 x^{12}+210 x^{8}\right) \\
& +\quad 4 \alpha\left(35525 x^{22}+41300 x^{18}+14934 x^{14}+2604 x^{10}+294 x^{6}\right) \\
& -\quad\left(166257 x^{24}+260750 x^{20}+220395 x^{16}+14756 x^{12}+1304 x^{8}+196 x^{4}\right),
\end{aligned}
$$

$$
\begin{aligned}
z_{2}= & 4096 \alpha^{6} \quad x^{28} \\
& -1024 \alpha^{5}\left(21 x^{30}+28 x^{26}\right) \\
& +256 \alpha^{4}\left(189 x^{32}+470 x^{28}+350 x^{24}\right) \\
& -\quad 64 \alpha^{3}\left(952 x^{34}+3192 x^{30}+4550 x^{26}+2576 x^{22}\right) \\
& +\quad 16 \alpha^{2}\left(2940 x^{36}+11200 x^{32}+21750 x^{28}+25452 x^{24}+12397 x^{20}\right) \\
& -\quad 4 \alpha\left(5733 x^{38}+22064 x^{34}+44324 x^{30}+82488 x^{26}+96761 x^{22}+40964 x^{18}\right) \\
& +\quad\left(7007 x^{40}+59388 x^{36}+35231 x^{32}+41132 x^{28}+278173 x^{24}+302918 x^{20}+94962 x^{16}\right)
\end{aligned}
$$

$$
\begin{aligned}
z_{3}= & 64 \alpha^{3} \quad x^{42} \\
& -16 \alpha^{2}\left(7 x^{44}+42 x^{40}\right) \\
& +4 \alpha\left(14 x^{46}+236 x^{42}+819 x^{38}\right) \\
& -\quad\left(7 x^{48}+308 x^{44}+4053 x^{40}+9842 x^{36}\right) .
\end{aligned}
$$

Pour rassembler tous mes résultats, je veux citer ceux que j'ai donné dans le Cambridge and Dublin Mathematical Journal [vol. II. (1847), 45, and vol. III. (1848), 57]. En écrivant la lettre $p$ au lieu de $n$ (symbole qui représente le carré du nombre $n$ de ce mémoire), et en changeant les signes des termes alternatifs, on aura pour solution particulière de l'équation

$$
p(p-1) x^{2} z+(p-1)\left(a x-2 x^{2}\right) \frac{d z}{d x}+\left(1-a x^{2}+x^{4}\right) \frac{d^{2} z}{d x^{2}}-2 p\left(\alpha^{2}-4\right) \frac{d z}{d \alpha}=0
$$

C. 
(savoir pour la solution qui pour $p=n^{2}$ se réduit au dénominateur de $\sin$ am $u$ ) la valeur

$$
z=1-C_{2} \frac{x^{4}}{1.2 .3 .4}+C_{6} \frac{x^{6}}{1.2 .3 \cdot 4 \cdot 5 \cdot 6}-\& c .
$$

les coefficients étant déterminés au moyen de l'expression

$$
\begin{aligned}
C_{r+2}=-(2 r+1)(2 r+2)(p-2 r)(p-2 r-1) C_{r}+(2 r+2)(p-2 r-2) \alpha C_{r+1} & \\
& -2 p\left(\alpha^{2}-4\right) \frac{d C_{r+1}}{d \alpha} .
\end{aligned}
$$

Cela donne les valeurs particulières suivantes:

$$
\begin{aligned}
& C_{2}=2 p(p-1) \\
& C_{3}=8 p(p-1)(p-4) \alpha \\
& \& c .
\end{aligned}
$$

[viz. with the change referred to, these are the values of $C_{2}, C_{3}, \ldots C_{8}$ given ante p. 299].

On remarquera que dans ces formules le premier terme de $C_{8}$ ne contient pas, comme on pourrait l'attendre, le facteur $(p-25)$. Cela vient de ce que le coefficient $C_{8}$ est composé des coefficients des termes correspondants de $z_{0}$ et $z_{1}$, tandis que les coefficients $C_{7}$, \&c., sont tout simplement des coefficients de $z_{0}$. La suite des coefficients $C$ offre plusieurs discontinuités de cette sorte. Par exemple on obtient généralement

$$
C_{r}=(-)^{r+1}\left\{\begin{aligned}
& 2^{2 r-3} p\left(p-1^{2}\right) \ldots\left(p-(r-1)^{2}\right) C_{r}{ }^{2} \alpha^{r-2} \\
+ & 2^{2 r-6} p\left(p-1^{2}\right) \ldots\left(p-(r-2)^{2}\right) C_{r}{ }^{2} \alpha^{r-4} \\
+ & 2^{2 r-9} p\left(p-1^{2}\right) \ldots\left(p-(r-3)^{2}\right) C_{r}{ }^{3} \alpha^{r-6} \\
+ & \& c .
\end{aligned}\right.
$$

mais le terme suivant ne contient pas le facteur $p\left(p-1^{2}\right) \ldots\left(p-(r-4)^{2}\right)$. Quant à la loi des coefficients $C_{r}^{1}, C_{r}^{2}, C_{r}^{3}$, on a

$$
\begin{aligned}
& C_{r}{ }^{1}=1 \\
& C_{r}{ }^{2}=(r-3)\{n(2 r-7)+(r-1)(8 r-7)\} \\
& C_{r}{ }^{3}=(r-4)(r-5)\left\{n^{2}\left(4 r^{2}-24 r+51\right)+n\left(32 r^{3}-220 r^{2}+412 r-255\right)\right. \\
& \left.\quad+2(r-1)(r-2)\left(32 r^{2}-88 r+51\right)\right\} .
\end{aligned}
$$

Également, en ordonnant la série suivant les puissances descendantes de $x$, la quantité $z$ étant la solution particulière qui pour $p=n^{2}$ ( $n$ impair) se réduit au dénominateur de $\sin$ am $n u$, on aura

$$
z=(-1)^{\frac{1}{2}(\sqrt{p-1)}} \cdot \sqrt{ } p\left(x^{p-1}-D_{1} \frac{x^{p-3}}{1.2 .3}+D_{2} \frac{x^{p-5}}{1.2 .3 .4 .5}-\& c .\right),
$$


où les coefficients $D$ sont donnés par l'expression

$$
\begin{aligned}
D_{r+2}= & -(2 r+3)(2 r+2)(p-2 r-2)(p-2 r-1) D_{r} \\
& +(2 r+3)(p-2 r-3) \alpha D_{r+1}-2 p\left(\alpha^{2}-4\right) \frac{d D_{r+1}}{d \alpha},
\end{aligned}
$$

ce qui donne les valeurs particulières suivantes:

$$
\begin{aligned}
& D_{1}=\quad(p-1) \alpha, \\
& D_{2}=2(p-1)(p+6) . \\
& \quad+(p-1)(p-9) \alpha^{2}, \\
& \text { \&c. }
\end{aligned}
$$

[viz. with the change referred to, these are the values of $D_{1}, D_{2}, \ldots D_{6}$ given ante pp. 364, 365].

Les mêmes remarques sont applicables aux coefficients $D$; seulement la discontinuité a lieu ici dès le coefficient $D_{4}$. Il paraît que c'est cause de cette discontinuité que le signe négatif se présente aux premiers termes des coefficients $D_{4}$, \&c. En effet, on a généralement:

$$
\begin{aligned}
D_{r}= & (p-1)(p-9) \ldots\left(p-(2 r-1)^{2}\right) \alpha^{r} \\
+ & (p-1)(p-9) \ldots\left(p-(2 r-3)^{2}\right) r(r-1)(p+4 r-2) \alpha^{r-2} \\
& +\& c .
\end{aligned}
$$

ici la discontinuité se présente déjà dans le terme suivant, qui ne contient pas le facteur $(p-1)(p-9) \ldots\left(p-(2 r-5)^{2}\right)$. Et c'est précisément le terme suivant qui devient négatif dans les expressions de $D_{4}, D_{5}$ et $D_{6}$. Mais tout cela est moins important que la théorie des séries partielles $z_{s}$, sur lesquelles les recherches ultérieures seront à fonder.

\section{PROBLÈME.}

Donner la solution de l'équation à différences

$$
\begin{gathered}
\left(-l \lambda-m \mu+(l-m)^{2}\right) P_{l, m} \\
+l(\lambda-2 l+2 m+2)(\lambda-2 l+2 m+1) P_{l-1, m} \\
+m(\mu+2 l-2 m+2)(\mu+2 l-2 m+1) P_{l, m-1} \\
-16 l m(\lambda \mu-(2 l+2 m-4)(\lambda+\mu)) P_{l-1, m-1}=0,
\end{gathered}
$$

dans laquelle $P_{0,0}=1$. (Voyez la "Note sur quelques formules \&c.") 